\title{
Pollution des eaux à usages domestiques dans les milieux urbains défavorisés des pays en développement : Synthèse bibliographique
}

\author{
Espérance O. HOUNSOUNOU ${ }^{1,2 *}$, Micheline AGASSOUNON DJIKPO TCHIBOZO ${ }^{1,2}$, \\ Nelly C. KELOME ${ }^{1}$, Expédit W. VISSIN ${ }^{1,3}$, Guy A. MENSAH ${ }^{4}$ et \\ Euloge AGBOSSOU ${ }^{1}$ \\ ${ }^{1}$ Institut National de l'Eau (INE), Université d'Abomey-Calavi (UAC), 01BP526 Cotonou, Bénin. \\ ${ }^{2}$ Laboratoire des Normes, de Contrôle de Qualité Microbiologique, Nutritionnelle et Pharmacologique, \\ Faculté des Sciences et Techniques (FAST), Université d'Abomey-Calavi (UAC), 01BP1636 RP Cotonou, \\ Bénin. \\ ${ }^{3}$ Laboratoire Pierre Pagney «Climats, Eau, Ecosystèmes et Développement 》, Faculté des Lettres, Arts et \\ Sciences Humaines, Université d'Abomey-Calavi, 03 BP 1122 Cotonou, Bénin. \\ ${ }^{4}$ Centre de Recherche Agricole d'Agonkanmey, 01 BP 2359 Recette Principale, Cotonou 01, Bénin. \\ *Auteurs correspondants ; E-mail : espolive89@yahoo.fr ; Tél :00(229) 67518537.
}

\section{RESUME}

Cette synthèse bibliographique sur l'état de la qualité des eaux à usages domestiques en milieux urbains de quelques pays africains a été faite à partir de la collecte des données issues des rapports d'activités de mémoires, de thèses, des publications et des ouvrages. Elle révèle que les différentes sources d'approvisionnement les plus utilisées (puits, forage, réseau public) sont confrontées à la pollution physique, chimique et microbiologique. Le niveau de pollution est dû aux germes de contamination fécale et pathogènes, des polluants azotés et les polluants métalliques toxiques. Les études consultées ont révélé que les eaux de puits sont plus exposées à la pollution. Quant aux eaux de forage et celles du réseau de distribution, elles sont moins vulnérables à la pollution. Les eaux, même étant potables à l'origine, peuvent subir une contamination le long de la chaîne d'approvisionnement. Les sources de pollution sont en général d'origines anthropiques. Les principaux facteurs de pollution identifiés ont été le manque d'assainissement de l'environnement et les conditions d'hygiène précaires des populations défavorisées des villes. Les conditions climatiques et physiques des milieux influencent le niveau de pollution des eaux. Le recours à ces eaux constitue une menace pour la santé des populations utilisatrices.

(C) 2016 International Formulae Group. All rights reserved.

Mots clés : eaux à usages domestiques, facteurs de pollution, polluants bactériologiques, polluants chimiques, contamination, santé humaine.

\section{Pollution of waters for domestic uses in the disfavored urban environments of the developing countries: Bibliographical synthesis}




\begin{abstract}
This bibliographical synthesis on the state of the quality of water for domestic use in urban environments of some African countries was made from the collection of data from the memories of activity reports, theses, publications and books. It reveals that the various water supply most used (well, drilling, public network) are confronted with the physical, chemical and microbiological pollution. The level of pollution is due to the germs of fecal contamination and pathogenic, the nitrogenized pollutants and the toxic metal pollutants. The consulted studies revealed that well waters are more exposed to the pollution. As for water of boring and those of distributing network, they are less vulnerable to pollution. Waters, even being drinkable originally, can undergo a contamination along the chain of supply. The sources of pollution are in general anthropic origins. The principal identified factors of pollution were the lack of sanitation of the environment and the precarious conditions of hygiene of the underprivileged populations of cities. The climatic and physical conditions of the environment influence the level of pollution waters. The recourse to this water constitutes a threat for public health users.

(C) 2016 International Formulae Group. All rights reserved.
\end{abstract}

Keywords: water for domestic uses, factors of pollution, bacteriological pollutants, chemical pollutants, contamination, human Health.

\section{INTRODUCTION}

$\mathrm{La}$ croissance démographique accompagnée d'une urbanisation rapide cause de nombreuses perturbations pour les milieux naturels (Ghazali et Zaid, 2013). Le milieu urbain concentre ainsi des populations et activités sur un territoire resserré, lequel doit supporter de nombreux flux (Host et TelleLamberton, 2014). Une grande partie de cette croissance se produit à l'intérieur ou autour des bidonvilles déjà surpeuplés, des zones périurbaines et des zones d'habitat informel. Des migrants ruraux d'une pauvreté extrême viennent s'installer dans des zones résidentielles dépourvues d'infrastructure élémentaire dédiée à l'eau et à l'assainissement (PNUD, 2006). C'est le cas dans la plupart des pays en développement où les problèmes majeurs auxquels les responsables communaux des grandes villes africaines doivent constamment faire face sont ceux liés à la gestion durable des déchets urbains et à l'accès à un système d'assainissement adéquat (Soro et al., 2010). La concentration de la population dans les zones urbaines ne fait qu'aggraver le problème d'accès à l'eau (Boubakar Hassane, 2010). Par ailleurs, l'industrialisation, l'utilisation non rationnelle des engrais et pesticides, ainsi que le manque de sensibilisation de la population pour la protection de l'environnement, conduisent autant à un déséquilibre de l'écosystème et génèrent des éléments polluants qui peuvent affecter la qualité physico-chimique et biologique des eaux (Mullis et al., 1997). Ces pollutions ne sont pas sans risques pour la population riveraine, avec la persistance de l'exposition aux polluants, les migrations possibles vers les eaux superficielles ou souterraines, responsables d'une dégradation de la ressource en eau potable (Host et TelleLamberton, 2014). Le problème affecte plus les populations vulnérables et démunies des quartiers défavorisés (Sy et al., 2011). Avec l'insuffisance du réseau conventionnel d'eau dans les milieux africains, ces populations font recours à des sources d'eau alternatives de qualité douteuse pour les besoins domestiques (Temgoua, 2011). Une eau non traitée peut contenir une multitude de parasites ou bactéries causant de graves maladies chez les hommes. Les pays en développement sont les plus touchés par ces problèmes de santé, car, faute de moyens, l'eau ne subit pas de traitement de purification avant d'être consommée (Alia, 2008). Par 
conséquent, près de deux millions d'habitants des pays pauvres meurent tous les ans suite à des maladies diarrhéiques dues à l'eau insalubre, à une mauvaise hygiène et à des installations sanitaires inadéquates (PNUD, 2006).

L'étude a pour objectif de mettre en exergue le niveau de pollution des eaux à usages domestiques dans les milieux urbains défavorisés des pays en développement à travers l'identification des polluants, et les principaux facteurs impliqués.

\section{SOURCES D'APPROVISIONNEMENT EN EAU DOMESTIQUE}

Une source d'approvisionnement en eau est un lieu ou un ouvrage à partir duquel le ménage s'approvisionne en eau pour son usage domestique quotidien (OMS/UNICEF, 2008). L'approvisionnement en eau est assuré par les eaux de surface (rivières, fleuves, lacs, mares, barrages), les eaux souterraines (puits, forages, sources) et les eaux de pluies (Aubry et Gaüzère, 2012).

\section{Eaux de surface}

Les eaux de surface ou superficielle regroupent les cours d'eau (rivières ou fleuves) et des retenues naturelles ou artificielles. Ces eaux de surface ont une qualité plus ou moins régulière selon les rejets qui s'y déversent ou encore selon le ruissellement des pluies (Coulibaly, 2005). Elles ont l'avantage de la quantité mais l'inconvénient majeur d'être fortement chargées en matières en suspension, voire en éléments pathogènes; ce qui implique des traitements souvent complexes et onéreux pour rendre l'eau potable pour des usages domestiques (pS-eau, 2012). Il ressort de la littérature que les eaux de surface ne sont consommées directement que dans les milieux ruraux en absence d'adduction d'eau publique ou lorsque les forages sont hors service. C'est le cas par exemple à Niakhar (Sénégal), et Oumako (Bénin) où les mares sont utilisés par les villageois soit pour la lessive, la vaisselle, la toilette, la cuisine ou la boisson (Chippaux et al., 2002 ; Yélognissè, 2007 ; Agassounon et al., 2014).

\section{Eaux souterraines}

Les eaux souterraines sont les ressources en eau privilégiées pour l'eau potable, car elles sont plus à l'abri des polluants que les eaux de surface (Guergazi et Achour, 2005). Elles sont les eaux des nappes, couches de terrains perméables saturés d'eau. La première nappe rencontrée sous le sol est la nappe phréatique située à moins de 50 mètres de profondeur et généralement séparées de la surface par quelques couches de terrains perméables. Il peut exister des nappes plus profondes, généralement captives (sous pression) situées à plus de 50 mètres de profondeur. Selon la profondeur de l'aquifère, les modes d'exploitation seront différents. Pour capter ces eaux, on utilise des puits ou des forages et des sources.

Le puits est l'ouvrage permettant de recueillir de l'eau grâce à un trou creusé dans le sol et ayant au moins $60 \mathrm{~cm}$ de diamètre et trois (3) mètres de profondeur pour capter l'eau d'une formation aquifère (Coulibaly et al., 2004). Les puits traditionnels ont des parois qui en général ne sont pas revêtues ou possèdent juste un revêtement peu épais de ciment non armé. Quant aux puits modernes à grands diamètres, leurs parois sont tenues par des buses en béton armé (Yélognissè, 2007). Le puits est en particulier adapté aux localités enclavées. Sauf exception, ce type d'ouvrages se destine aux nappes phréatiques. L'exhaure peut être assurée par puisage manuel (seau et corde), par pompe à corde (nappe peu profonde) ou par pompe à motricité humaine (nappe d'eau de profondeur supérieure à 10 mètres) (Molinie, 2009).

Le forage est un ouvrage de captage de l'eau souterraine de petit diamètre (en général 15 à 40 centimètres). Le forage est équipé d'un système mécanique ou électromécanique 
d'exhaure dans les aquifères profonds (pSEau, 2012).

Contrairement aux puits et aux forages, les sources ne nécessitent aucun système d'exhaure. L'aménagement consiste en la réalisation d'un ouvrage de génie civil permettant de collecter et de rassembler différents filets d'eau, éventuellement à l'aide de drains avec l'aire de captage qui doit être soigneusement protégée (pS-Eau, 2012).

Les différents types d'approvisionnement en eau souterraine décrits ci-dessus (puits, forages et sources aménagées) constituent les moyens courants de desserte en eau de consommation en cas de déficit du réseau d'eau potable dans la plupart des pays en développement (Odoulami, 2009 ; Degbey et al., 2010 ; Ahoudi et al., 2015; Soncy et al., 2015).

\section{Eaux de réseau de distribution publique}

Elles sont produites à partir des eaux souterraines provenant de sources ou de forages, soit des eaux superficielles provenant de pompage direct dans des cours d'eau, des canaux, des lacs ou des étangs. Après traitement, elles sont envoyées dans le réseau de distribution pour arriver au robinet des consommateurs. Les points de distribution de l'eau comprennent les robinets publics (bornefontaine) relevant d'investissements publics et des robinets domiciliaires (branchements privés) installés par les ménages (pS-eau, 2012). Les populations qui résident dans les quartiers précaires à bas revenus n'ont pas les moyens d'installer ces branchements à domicile (Mpakam et al., 2006).

L'eau de distribution est le « produit alimentaire » le plus contrôlé. Sa qualité est en général satisfaisante et répond à des exigences sanitaires réglementaires. Au niveau national, ce sont les services publics qui se chargent de la distribution de l'eau potable. A titre d'exemple, en Centrafrique c'est la Société de Distribution des Eaux de Centrafrique (SODECA) qui en a la charge (Nguimal al.,
2005); au Cameroun, c'est la société Nationale des Eaux du Cameroun (SNEC) (Mpakam et al., 2006) et au Bénin, la Société Nationale des Eaux du Bénin (SONEB) s'en occupe (Agassounon et al., 2015)

\section{Eaux de pluie}

L'eau de pluie représente une option parallèle à d'autres technologies d'approvisionnement en eau, surtout dans les zones rurales, mais également de plus en plus dans les zones urbaines. Les eaux de pluie peuvent être collectées à partir des toitures des maisons dans des citernes. Pour le stockage de l'eau à très petite échelle, dans les pays en de développement, des cuvettes, des seaux en plastique, des pots en terre ou en céramique, de vieux barils de pétrole ou des récipients alimentaires vides sont utilisés. C'est une pratique qui existe depuis des siècles (Worm et Hattum, 2006). La collecte et le stockage de l'eau de pluie fournit de l'eau pour les usages domestiques dans les périodes de pénurie, mais aussi pendant la saison des pluies. Cependant, ce système a quelques inconvénients liés à la qualité de l'eau qui peut être affectée par la pollution de l'air, les déjections d'animaux ou d'oiseaux, des insectes, de la saleté et de la matière organique en absence de l'entretien des toitures. L'approvisionnement en eau est mis en cause par de longues périodes sécheresses (Worm et Hattum, 2006). Alors, le recours à l'eau de pluie est une pratique non permanente ou provisoire d'approvisionnement en eau domestique.

\section{PARAMETRES DE QUALITE DES EAUX DE CONSOMMATION}

L'eau potable doit convenir à la consommation humaine et pour tous les usages domestiques habituels (OMS, 1997). Elle doit répondre aux normes de potabilité proposées au niveau mondial et reprises localement par des organismes nationaux. $\mathrm{La}$ qualité d'une eau est caractérisée par les 
diverses substances qu'elle contient, leur quantité et l'effet qu'elles ont sur l'écosystème et sur l'être humain (Festy et al., 2003).

\section{Contaminants microbiologiques, indicateurs de pollution et effets sanitaires}

Les principales catégories de microorganismes pathogènes d'origine hydrique sont les bactéries, les virus et les protozoaires. L'évaluation pratique de la qualité microbiologique des eaux se fait sur la base d'organismes dits « indicateurs » (Festy et al., 2003 : Servais et al., 2009 ) ; choix qui résulte des difficultés ou impossibilité technique de détecter toute la diversité des microorganismes (Givord et Dorioz, 2010). Les bactéries indicatrices de contamination fécale les plus appropriées sont les Coliformes connus sous le nom de Escherichia coli et Entérocoques fécaux ou intestinaux (Rodier et al., 2009). En plus de ces bactéries, d'autres auteurs (Garcia-Armisen, 2006 ; Rodier et al., 2009) ont mentionné l'utilisation des Coliformes totaux, Coliformes thermotolérants et les Anaérobies sulfitoréducteurs notamment Clostridium perfringens et les bactéries aérobies revivifiables comme indicateurs de pollution et d'efficacité de traitement.

Les microorganismes pathogènes les plus fréquemment rencontrés dans les eaux sont consignés dans le Tableau1, ainsi que les pathologies dont ils sont responsables. Les microorganismes pathogènes présents dans l'eau sont le plus souvent transmis par voie féco-orale (Servais et al., 2009; Hartemann, 2013), et la contamination de l'homme se réalise alors soit par consommation d'eau de boisson, soit par consommation d'aliments contaminés par l'eau, soit encore lors d'un bain ou d'un contact avec des eaux à usage récréatif (Servais et al., 2009; Aubry et Gaüzère, 2012). En fonction des voies de transmission, on distingue les maladies hydriques (liées à l'eau de boisson) telles que la fièvre typhoïde, hépatite, choléra, amibiase, giardiase, ...) ; les maladies liées aux pénuries d'eau en terme de défaut de l'hygiène (cécité des rivières, gale, mycoses, poux...); les maladies aquatiques liées aux vers (helminthiases) et les maladies dues aux vecteurs tels les insectes (Paludisme) (Rosillon, 2003).

\section{Contaminants physicochimiques des eaux et effets sanitaires}

Ils regroupent les paramètres physicochimiques liés à la structure naturelle de l'eau, les éléments indésirables et ceux toxiques (Vaute, 1999). Les paramètres physicochimiques liés à la structure naturelle de l'eau incluent la température, le $\mathrm{pH}$, la conductivité, les ions majeurs: le bicarbonate, le chlorure, le sulfate, le calcium, le magnésium, le sodium, le potassium et autres, (Burnol et al., 2006). Les éléments indésirables prennent en compte les composés azotés (ammonium, nitrite, nitrate), phosphatés (phosphate); les composés organiques (hydrocarbures totaux, l'indice phénol); les métaux et autres éléments (argent, bore, barium, cuivre, fluor, fer, manganèse et zinc). Les éléments toxiques comprennent l'arsenic, le cadmium, le chrome, le nickel, le plomb et le mercure; ils constituent les éléments traces métalliques et sont généralement définis comme des métaux lourds. Trois de ces métaux (cadmium, plomb, mercure) ont été identifiés comme des "substances dangereuses prioritaires", et sont soumises à un objectif de rejet zéro dans les eaux souterraines dans la Décision 2455/2001/CE du conseil Européen modifiant la Directive Européenne sur l'Eau 2000/60/CE (Burnol et al., 2006).

A l'opposé du risque microbiologique qui s'exerce à court terme, le risque chimique est essentiellement à moyen et long terme (Payment et Hartemann, 1998). L'origine (Tableau 2) et l'effet sanitaire des éléments chimiques (Tableau 3) de quelques éléments chimiques indésirables sont décrits ci-dessous. 


\section{QUALITE DES EAUX A USAGES DOMESTIQUES EN MILIEUX URBAINS}

Plusieurs études récentes se sont penchées sur les questions liées à la qualité de l'eau utilisée (puits, forages et sources, réseau public) par les populations africaines pour les diverses usages domestiques en milieu urbain. Ces études ont mis en évidence le niveau de pollution des eaux par les microorganismes (germes de contamination fécale et ceux pathogènes), les éléments chimiques indésirables et les métaux lourds. Ces études ont permis d'identifier les sources potentielles de pollution et les moyens de remédiation.

\section{Qualité microbiologique des eaux}

Diverses études ont mis en évidence la pollution des eaux par les microorganismes.

Les études réalisées par Djuikom et al. (2009); Mbawala et al. (2010); Benajiba et al. (2013) ; Soncy (2015); Hounsinou et al. (2015) ; Zerhouni et al. (2015) respectivement à Douala (Cameroun), Ngaoundéré (Cameroun), Martil (Maroc); Lomé (Togo); Abomey-calavi (Bénin) et Sebaa Ayoune (Maroc) ont révélé la contamination fécale par la présence individuelle ou associée des Coliformes totaux, des Coliformes thermotolérants, des Entérocoques fécaux et du Clostridium perfringens dans les eaux de puits. La flore totale y a aussi été dénombrée (Bricha et al., 2007 ; Soncy et al., 2015) . Ces eaux hébergent également de nombreux germes pathogènes parmi lesquelles figurent les bactéries telles que Salmonella sp, Vibrio parahaemolyticus, Pseudomonas aeruginosa (Mbawala et al., 2010) ; Shigella spp, Klebsiella pneumoniae, Staphylococcus aureus et Candida albicans (Degbey et al., 2010) ; Proteus penneri, Stenotrophomonas maltophilia, Citrobacter freundii (Nanga et al., 2014) et les parasites tels que (kystes de Entamoeba histolytica et de Giardia lamblia, œufs de Cryptosporidium et de Ascaris lombricoïdes (Mbawala et al., 2010). Les eaux de puits sont de qualité microbienne plus défectueuse que celle des eaux de forage (Chippaux et al., 2002; Dovonou, 2012; Soncy et al., 2015), de source (Ghazali et Zaid, 2013) et de distribution publique (El Ouedghiri et al., 2014 ; Mahamat et al., 2015). Dovonou (2012) et Soncy et al. (2015) ont trouvé une différence significative entre les eaux de puits et celles de forage étudiées par rapport à tous les germes recherchés. L'étude de Agassounon et al. (2015) sur la flore reviviscente du réseau de distribution de la Société Nationale des Eaux du Bénin (SONEB) de Savalou a révélé une présence de la flore aérobie revivifiable et de Pseudomonas aeruginosa. En Centrafrique, Nguimal et al. (2005) ont identifié la présence des Coliformes, de la flore totale, des levures, des moisissures dans les eaux de distribution. L'étude de Ouhsassi et al. (2013) a mis en évidence les Coliformes, les Streptocoques, les Anaérobies sulfitoréducteurs et les Staphylocoques, Salmonella et Campylobacter dans les eaux du réseau public de Medina de Tétouan au Maroc.

\section{Qualité physico-chimique des eaux}

Les études récentes réalisées sur la qualité chimique des eaux à usages domestique en milieux urbains ont montré que l'état de pollution de ces eaux varie d'une région à une autre. En effet, l'étude de Bricha et al. (2007) sur sept (7) point d'eaux de puits utilisés à des fins alimentaires à M'nasra (Maroc) a montré une forte concentration en nitrates (5 fois plus élevé que celle recommandée $(50 \mathrm{mg} / \mathrm{l})$ pour la consommation. Au Sud-Ouest de Brazzaville (Congo), l'analyse de la qualité physicochimique des eaux souterraines (27 puits et 1 source) utilisées pour la boisson a donné des concentrations en ions majeurs $\left(\mathrm{Ca}^{2+}, \mathrm{Mg}^{2+}\right.$, $\left.\mathrm{K}^{+}, \mathrm{Cl}^{-}, \mathrm{SO}^{2-}\right)$ toutes inférieures aux valeurs guide de l'OMS. Le paramètre le plus hors norme a été le chrome VI (Matini et al., 2009). En Côte d'ivoire, l'analyse de la 
qualité des eaux de puits du département d'Abengourou par Aka et al. (2013) a montré que les paramètres physico-chimiques les plus déclassant en rapport avec les valeurs guides de l'OMS pour la consommation sont le $\mathrm{pH}$, le Fer, l'aluminium et les nitrates. Au Togo, la caractérisation physico-chimique des eaux (26 puits et de 4 forages) du quartier Agoê-zongo par Ahoudi et al. (2015) a révélé également des teneurs en nitrates allant jusqu'à 9 fois la norme de l'OMS et des teneurs en plomb, cadmium, et nickel allant respectivement jusqu'à 78,$26 ; 42,23$; et 15,45 fois les normes de l'OMS. Les teneurs en potassium et sodium vont respectivement jusqu'à 9 fois et 1,8 fois les normes françaises. Au Bénin, Hoteyi et al. (2014) ont déterminé une pollution azotée (concentration en nitrates variant de 75 à $97 \mathrm{mg} / \mathrm{l})$ des eaux de puits utilisées pour la production de l'eau en sachet dans la ville de Porto-Novo. De même, les eaux de cent dix (110) puits étudiées par Degbey et al. (2009) à Abomey-calavi ont présenté une forte pollution en nitrates $(32,73 \%)$, en nitrites $(11,82 \%)$, en aluminium $(10 \%)$, en fer $(43,64 \%)$ total, en cadmium $(6,36 \%)$ et en mercure $(14,55 \%)$. Dans la même ville, les études de Hounsinou et al. (2015) sur les eaux de (20) puits ont donné des taux de conformité aux normes de l'OMS de $40 \%(\mathrm{pH}), 60 \%$ (conductivité électrique), $65 \%$ (turbidité) et $100 \%$ (zinc). Dans la commune de Pobè, les travaux de Lagnika et al. (2014) ont aussi identifié une pollution azotée se traduisant par des taux élevés en nitrates et en ammonium dans plus de $50 \%$ des douze (12) puits étudiés.

Contrairement aux eaux de puits, celles de distribution publique et de forages sont moins polluées chimiquement. Au Tchad, une étude comparée de la qualité a montré que les eaux traitées (de distribution publique) sont de bonne qualité physico-chimique que celle de forage (Mahamat et al., 2015). Cependant, Nguimal et al. (2005) ont constaté une détérioration de la qualité physique (augmentation de la turbidité et de la couleur, réduction du chlore résiduel) des eaux de distribution à Bangui.

\section{Dégradation de la qualité de l'eau le long de la chaîne de l'eau}

En l'absence de réseau de canalisation, l'approvisionnement en eau impose un déplacement régulier des femmes entre leur habitation et la source la plus proche. Ce type d'approvisionnement fait intervenir la notion de «chaîne de l'eau» constituée d'une phase de transport et de stockage (Lalane, 2012). Pour suivre l'état de contamination de ces eaux, le long de la chaîne, des études ont été effectuées sur la caractérisation de leur qualité physique, chimique et bactériologique. Les travaux de Ngnikam et al. (2007) à Yaoundé (Cameroun) sur les eaux issues du réseau d'eau potable conservées dans 26 ménages ont révélé que l'eau a subi une dégradation importante de sa qualité par la présence des streptocoques et des coliformes thermotolérants. De même, les études de l'UNICEF (2011) en Mauritanie sur les eaux de robinet et celles de Lalanne (2012) et Nkurunziza (2013) au Burkina Faso sur les eaux de forage ont aussi détecté la contamination microbiologique le long de la chaîne d'utilisation. Sur le plan physicochimique la qualité de l'eau des sources est bonne et se conserve le long de la chaîne de l'eau (Lalanne, 2012). Cependant, cet auteur a pu observer pendant la phase de transport une légère augmentation de la température de l'eau, puis après le stockage une augmentation du $\mathrm{pH}$ et de la turbidité et une diminution de la température. De pareils constats ont été faits par Lavie et El-Tayib (2014) dans la sphère domestique de la zone périphérique de Khartoum au Soudan. Les germes à l'origine de la contamination ont été les streptocoques fécaux, les coliformes totaux et les coliformes thermotolérants. Les principales causes de contamination sont entre autres les comportements des usagers et les conditions de transport et de stockage de l'eau (Ngnikam 
et al., 2007; Ndiaye et al., 2010; Sackou Kouakou et al., 2010 ; Lalane, 2011).

\section{Origine et facteurs de pollution des eaux à usage domestiques}

L'origine de la pollution des eaux peut être naturelle ou anthropique (Laurent, 2013). Les principaux facteurs qui contrôlent la qualité physico-chimique et bactériologique des eaux sont les activités anthropiques, le contexte hydrogéologique et le climat, (Van der Werf, 1996 ; Mfonka et al., 2015).

Les principales sources de pollution anthropique sont l'agriculture, qui s'applique de manière diffuse sur le territoire; les industries qui sont à l'origine de rejets très diversifiés et souvent localisés et les activités domestiques via les rejets d'eaux usées ou les décharges (Kenfaoui, 2008; Laurent, 2013). $\mathrm{La}$ nature des polluants dépend de l'occupation du sol et des activités du milieu considéré (Zgheib, 2009). Plusieurs auteurs ont relevé les facteurs tels que les activités autour des puits, le défaut d'aménagement des puits, la proximité des sources de pollution telles les déchets urbains, décharges sauvages d'ordures, les ouvrages d'assainissement individuel (latrines, puisards, Fosses septiques), et le non-respect des règles d'hygiène élémentaire par les utilisateurs à l'origine de la pollution (microbienne et/ou chimique) des eaux de puits en milieux urbains (Sagbo, 1999; Kessou, 2000; Chippaux et al., 2002; Djuikom et al., 2009; Mpakam et al., 2009 ; Mbawala et al., 2010; Yapo et al., 2010; Degbey et al., 2011; Dovonou, 2012; Belghiti et al., 2013 ; Zerhouni et al., 2015). La pollution organique peut être liée à l'infiltration des eaux usées et des engrais chimiques (El Asslouj et al., 2007 ; Lagnika et al., 2014 ). Le lixiviat des décharges publiques constitue le principal vecteur de transport de la pollution métallique issue des sites de stockage des déchets vers la nappe phréatique (Jourdan et al., 2005; Er-Raioui et al., 2011).

Outre l'action humaine, les propriétés physiques du milieu et le contexte climatique sont des facteurs déterminants de la pollution des eaux. Par rapport à l'influence des conditions climatiques sur la pollution des eaux, la variation saisonnière a été le facteur le plus mentionné dans la littérature. En effet, la saison d'échantillonnage influe sur les concentrations chimique et/ou microbienne des eaux de la nappe phréatique (Bricha et al., 2007 ; Benajiba et al., 2013, Mpakam et al., 2009; Hounsinou et al., 2015). Certains auteurs ont estimé une augmentation du taux de contamination pendant la saison sèche par rapport à la période pluvieuse (Boubakar Hassane, 2010 ; Benajiba et al., 2013 ). L'élévation de la température et le processus évaporatoire pendant la saison sèche peuvent aussi justifier cette augmentation (Boubakar Hassane, 2010). Les conditions pluviales peuvent avoir un rôle de dilution dans la nappe phréatique et par conséquent diminuer la concentration bactérienne dans l'eau pendant la saison pluvieuse (Bahir et al., 2002 ; Lallahem, 2002). Par contre d'autres travaux (Bricha et al., 2007 ; Hounsinou et al., 2015) ont montré que la qualité bactériologique et/ou chimique des eaux souterraines s'est plus détériorée en saison des pluies. Pendant la période hivernale ou pluvieuse, l'augmentation du niveau piézométrique accélère la propagation des polluants qui se trouvent sur le sol ou piégés dans la zone insaturée (Bricha et al., 2007). La pluie réduit la rétention des microorganismes par le sol par l'abaissement de la concentration ionique et l'augmentation de la vitesse d'infiltration du sol (Bitton et Gerba, 1984). Les eaux d'infiltration favorisent la pollution par les fosses septiques, les latrines non étanches (Hounsinou et al., 2015) qui se trouvent en intercommunication avec les puits (Mpakam et al., 2009).

Les propriétés intrinsèques ou le contexte géologique et hydrogéologique des milieux traversés, contrôlent la migration des polluants (Van der Werf, 1996 ). Le risque de pollution chimique et biologique dépendent d'une part des caractéristiques des eaux d'infiltration, et d'autre part, des propriétés 
naturelles des couches géologiques qui séparent la nappe phréatique de la surface du sol (Nola et al., 2006). Le risque de contamination est plus important avec la perméabilité du sol (ex : sablonneux) recouvrant la nappe, et la faible profondeur de la nappe par rapport à la surface (Degbey et al., 2010 ; El Ouedghiri et al., 2014).

Par ailleurs plusieurs facteus peuvent être responsables de la contamination de l'eau $\mathrm{du}$ réseau public notamment le défaut d'étanchéité des tuyaux de distribution (Ndiaye et al., 2010); incidents survenant (réparations, branchements, fuites....) sur le réseau (Gauthier, 2002 ; Hartemann, 2013) et la présence de biofilms dans les réseaux de distribution d'eau (Gauthier, 2002 ; Poisson, 2011).

\section{Moyens de remédiation aux risques liés aux pollutions des eaux de consommation}

Face aux problèmes de pollution des eaux de consommation et les risques sanitaires associés, plusieurs recommandations ont été faites dans la littérature pour y remédier. Sans être exhaustives, les principales recommandations ont porté sur la sensibilisation des populations concernées sur les maladies du péril hydrique liées aux manquements aux mesures d'aménagement, d'assainissement et d'hygiène appropriées (Agassounon et al., 2012); l'éducation aux bonnes pratiques pour le changement de comportements (Ngnikam et al., 2007 ; Totin et al., 2008 ; Ndiaye et al., 2010); la gestion des déchets, la mise en place des ouvrages d'assainissement adaptés au contexte socioéconomique et géographique (Mpakam et al., 2009) ; l'équipement des villes en système d'assainissement liquide, l'interdiction de rejets anarchiques des eaux usées domestiques et industrielles (Benajiba et al., 2013). La surveillance et la désinfection régulières des eaux souterraines ont été aussi suggérées pour réduire le degré de contamination bactériologique des eaux polluées avant leur utilisation (Djuikom et al., 2009 ; Benajiba et al., 2013 ; Soncy et al., 2015).

Ngnikam al. (2007) au Cameroun, Nkurunziza (2011) et Lalanne (2012) au Burkina Faso ont procédé à l'éducation et à la sensibilisation des populations pour impulser le changement de comportement après avoir détecté la détérioration de l'eau de boisson sur la chaîne d'approvisionnement. Dovonou (2012) après avoir démontré la vulnérabilité de l'aquifère superficiel du champ de captage de Godomey (Bénin) a mis en place un plan de délimitation des aires de protection pour une gestion durable des ressources en eau exploitées. Fatombi et al. (2012) et Kaboré et al. (2013), respectivement, au Bénin (Lagbé) et au Burkina Faso (Ouagadougou et Kadiogo) ont essayé le traitement des eaux souterraines et des eaux de surface contaminées par diverses bactéries et polluants chimiques avec Moringa oleifera avec l'obtention d'une nette amélioration de la qualité de l'eau. Au Brazzaville (Congo), Andzi Barhé et Bouaka (2013) après avoir effectué des essais de désinfection des eaux de puits avec de l'hypochlorite de calcium, ont recommandé l'association d'autres techniques pour éviter la formation des composés organohalogénés. Pour permettre un traitement des eaux de puits en continu, Hoteyi et al. (2014) ont mis en place un pot diffuseur qui est en fait un dispositif qui assure la chloration continue de l'eau en libérant progressivement une quantité nécessaire à la destruction des microorganismes.

Les différentes recommandations faites dans la littérature doivent être contextualisées selon les facteurs de pollution en jeu (Tableau 4). Pour une meilleure résolution des problèmes de qualité des eaux de consommation dans une zone donnée, les études doivent passer par l'identification des principales causes de pollution, la caractérisation des polluants et apporter des solutions adéquates. 
Tableau 1 : Principaux groupes et genres d'agents pathogènes responsables de maladies d'origine hydrique (Servais et al., 2009).

\begin{tabular}{|c|c|c|}
\hline Groupes de microorganismes & Pathogènes & Pathologies \\
\hline \multirow[t]{7}{*}{ Virus } & $\begin{array}{c}\text { Entérovirus } \\
\text { (polio, écho, coxsackie) } \\
\text { Hépatite A et E }\end{array}$ & $\begin{array}{c}\text { Méningite, paralysie, fièvres, myocardie, problèmes } \\
\text { respiratoires et diarrhée } \\
\text { Infections hépatiques }\end{array}$ \\
\hline & Norovirus & Diarrhée/ gastro-entérite \\
\hline & Sapporovirus & Diarrhée/ gastro-entérite \\
\hline & Rotavirus & Diarrhée/ gastro-entérite \\
\hline & Astro virus & Diarrhée \\
\hline & Adenovirus & Diarrhée, infections oculaires et problèmes respiratoires \\
\hline & Reovirus & Problèmes respiratoires et entériques \\
\hline \multirow[t]{6}{*}{ Bactéries } & Salmonella & Fièvre typhoïde et diarrhée \\
\hline & Shigelia & Diarrhée \\
\hline & Campylobacter & Diarrhée (cause première des intoxications alimentaires) \\
\hline & Yersinia enterolitica & Diarrhée \\
\hline & $\begin{array}{c}\text { Escherichia coli } O 157: H 7 \text { et d'autres } \\
\text { souches }\end{array}$ & $\begin{array}{l}\text { Diarrhée risque de complications (urémie hémolytique) chez } \\
\text { les enfants en bas âges }\end{array}$ \\
\hline & Legionella pneumophila & Pneumonie et autres infections respiratoires \\
\hline \multirow[t]{2}{*}{ Protozoaires } & $\begin{array}{l}\text { Naegleria fowleri } \\
\text { Naegleria fowleri }\end{array}$ & Méningo-encéphalite \\
\hline & Entomoeba histolytica & Dysenterie amibienne \\
\hline
\end{tabular}


E. O. HOUNSOUNOU et al. / Int. J. Biol. Chem. Sci. 10(5): 2392-2412, 2016

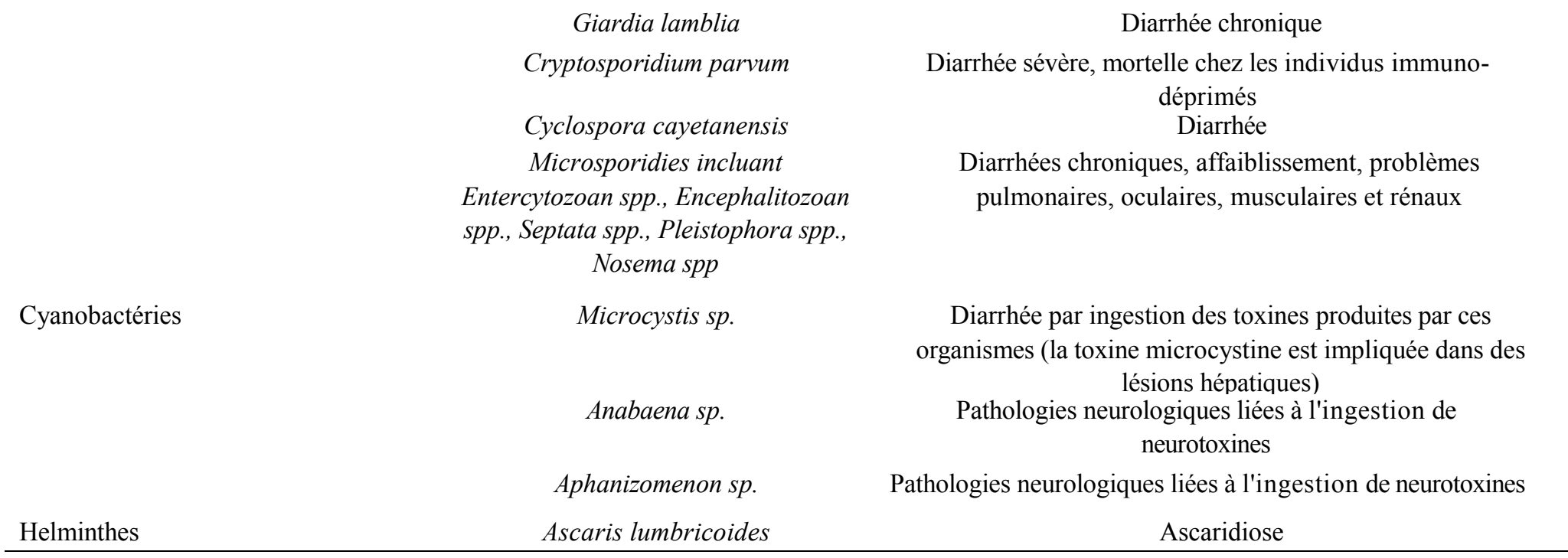


Tableau 2 : Quelques paramètres chimiques indésirables et leur source.

\begin{tabular}{|c|c|}
\hline Paramètres & Sources \\
\hline Nitrates et Nitrites & $\begin{array}{l}\text { Activités humaines (santé Canada, 1992); installations septiques déficientes (Levallois et } \\
\text { Phaneuf, 1994); engrais, eaux usées, décharges, eaux de ruissellement, lessivage des terres par } \\
\text { les précipitations et l'oxydation de l'azote, oxydation des nitrites par les bactéries suite à } \\
\text { l'infiltration des eaux usées ; matières fécales des animaux à sang chaud, fosses d'aisance à fond } \\
\text { perdu (Mfonka et al., 2015). }\end{array}$ \\
\hline Phosphates & activités agricoles et rejets urbains, (Hartemann, 2013). \\
\hline Plomb & $\begin{array}{l}\text { Métallurgie (monnaie, canalisations et ustensiles de cuisine), fabrication des peintures et } \\
\text { d'hydrocarbures ; formation des aérosols plombiers issus de la fusion de minerais, utilisation de } \\
\text { combustibles fossiles (Ahonon, 2011); corrosion des composantes structurales des réseaux de } \\
\text { distribution et des tuyauteries domestiques en présence d'oxydants (oxygène dissous et Chlore) } \\
\text { (Viraraghavan et al., 1999). }\end{array}$ \\
\hline Mercure & $\begin{array}{l}\text { Activité volcanique, dégazage des sols ; sources anthropogéniques : industries de production de } \\
\text { l'électricité par combustion (charbon, mazout, etc.), de fusion des métaux et d'incinération de } \\
\text { matières résiduelles ; objets d'usage courant : tubes fluorescents, interrupteurs à bascule, produits } \\
\text { des technologies de l'information et des télécommunications, les thermomètres domestiques et les } \\
\text { thermostats ainsi que l'amalgame dentaire (Gendron et al. 2007). }\end{array}$ \\
\hline Cadmium & $\begin{array}{l}\text { Erosion naturelle, lessivage des sols décharges industrielles (Botta et Bellon, 2004) ; } \\
\text { solubilisation par les acides des substances alimentaires à partir de poteries vernissées, de boîtes } \\
\text { de conserve et d'ustensiles de cuisine galvanisés (Rodier et al., 2009). }\end{array}$ \\
\hline
\end{tabular}




\begin{tabular}{ll}
\hline Arsenic & Rejets d'eaux résiduaires industrielles, traitement de minerais arsenicaux (cuivre...), combustion \\
& du charbon ou de déchets, dépôts de résidus industriels, utilisation d'engrais phosphatés, \\
& d'herbicides, d'insecticides et de détergents (les eaux de blanchisserie) érosion naturelle, \\
& $\begin{array}{l}\text { lessivage des particules telluriques, éruptions volcaniques, zones de fractures sous-marines } \\
\text { (Rodier et al., 2009), }\end{array}$ \\
\hline Chrome & Teintures, colorants et rejets des ateliers de galvanoplastie (Rodier et al., 2009) \\
\hline Argent & $\begin{array}{l}\text { Minerais (un sous-produit de l'extraction du cuivre, du plomb et du zinc); bijouterie, miroiterie, } \\
\text { photographie, métallurgie, contacts électriques, amalgames dentaires et médecine (collyres, } \\
\text { collutoires) (Rodier et al., 2009). }\end{array}$ \\
\hline Aluminium & Métallurgie, constructions automobile et aéronautique, fabrication d'appareils ménagers (Botta et \\
& Bellon, 2004). \\
\hline Pesticides & Entrainement des insecticides, fongicides, herbicides, corvicides, molluscicides, rodenticides et \\
& acaricides par les eaux de pluie et de ruissellement vers les cours d'eau et nappe phréatique (Botta \\
& et Bellon, 2004).
\end{tabular}

Tableau 3 : Effets sanitaires liés à quelques paramètres chimiques indésirables de l'eau.

\begin{tabular}{ll}
\hline Paramètres & Effets sanitaires \\
Nitrates, & -Méthémoglobinémie résultant de la réduction des nitrates en nitrites par les microorganismes \\
Nitrites & empêchant l'hémoglobine de transporter de l'oxygène (Association Santé Environnement France, \\
& 2010) \\
& -Effet cancérigène résultant des nitrosamines (composés formés à partir de la combinaison des \\
& nitrites avec certaines amines ou amides (Botta et Bellon, 2004)
\end{tabular}



histopathologiques variées (Degbey et al., 2010) ; atteintes cognitives et neurocomportementales (StLaurent et al., 2013); augmentation de la sensibilité aux effets toxiques du plomb chez les jeunes enfants liée à la perméabilité de la barrière hématoencéphalique; l'anorexie, vomissements, irritabilité, troubles de comportement ; chez la femme enceinte, nuisance au développement du système nerveux central du fœtus; chez l'adulte : douleurs abdominales (colique) et constipation; intoxication sévère :convulsions, coma, mort (INSPQ, 2003).

Mercure L'intoxication par les sels de mercure (hydrargyrisme), caractérisée par des lésions des centres nerveux se traduisant par des tremblements. Insuffisance rénale irréversible due aux effets à long terme des dérivés minéraux du mercure sur les reins et effet des dérivés organiques (organomercuriels) sur le système nerveux central (Botta et Bellon, 2004).

Cadmium Troubles rénaux, d'altérations osseuses et d'hypertension artérielle ; « Itai Itai » caractérisé par une décalcification osseuse, une protéinurie et une glucosurie (Rodier et al., 2009).

Arsenic Intoxication aiguë : troubles gastro-intestinaux plus ou moins accentués, troubles hépatiques et rénaux et manifestations cardiovasculaires; hypertension et tachycardie; intoxication chronique : troubles digestifs, syndromes mélano-dermiques et neurologiques (Rodier et al., 2009).

Chrome Intoxication aiguë: tubulonéphrite parfois compliquée d'une hépatite toxique avec ictère ; intoxication chronique: lésions cutanées et des muqueuses avec des atteintes de l'appareil respiratoire (bronchites, asthme, cancers broncho-pulmonaires) (Rodier et al., 2009).

Argent Troubles digestifs, un liseré gingival et une coloration gris bleuâtre de la peau. Cette dyschromie prédomine sur le visage et les partie découvertes, ainsi que sur les ongles (Rodier et al., 2009).

Aluminium Maladie d'Alzheimer et syndromes parkinsoniens (Botta et Bellon, 2004).

Pesticides Effets reprotoxiques (malformations, stérilité, troubles de la reproduction), mutagènes et cancérogènes (Botta et Bellon, 2004 ; Association Santé Environnement France, 2010). 
Tableau 4 : Liste de quelques facteurs de pollution de l'eau et approches de solutions.

\begin{tabular}{lll}
\hline Facteurs de pollution & \multicolumn{1}{l}{ Approches de solutions } \\
\hline $\begin{array}{l}\text { Rejets anarchiques des déchets liquides (eaux } \\
\text { usées) }\end{array}$ & $\begin{array}{l}\text { L'installation des ouvrages d'assainissement de } \\
\text { base améliorés }\end{array}$ \\
\hline $\begin{array}{l}\text { Mauvaise gestion des déchets solides et } \\
\text { installation des décharges sauvages }\end{array}$ & $\begin{array}{l}\text { La gestion hygiénique des déchets solides par la } \\
\text { collecte, le traitement, l'élimination et/ ou le } \\
\text { recyclage des déchets }\end{array}$ \\
\hline $\begin{array}{l}\text { Mauvaise évacuation des excréments humains et } \\
\text { animaux }\end{array}$ & $\begin{array}{l}\text { L'évacuation hygiénique des excréments à } \\
\text { travers la construction et l'utilisation } \\
\text { correcte des installations sanitaires appropriées }\end{array}$ \\
\hline $\begin{array}{l}\text { Défaut d'aménagement des points d'eau } \\
\text { (conditions hydrogéologiques) }\end{array}$ & $\begin{array}{l}\text { La protection et l'aménagement adéquats des } \\
\text { points d'eau (sources, puits, forage) }\end{array}$ \\
& & \\
\hline $\begin{array}{l}\text { Mauvaises conditions de transport et de } \\
\text { stockage de l'eau dans les domiciles }\end{array}$ & $\begin{array}{l}\text { La propreté et la protection des récipients de } \\
\text { collecte, de stockage, et de prélèvement de } \\
\text { l'eau; hygiène personnelle (lavage des mains au } \\
\text { savon et eau en temps convenus) et hygiène de } \\
\text { la sphère domestique }\end{array}$ \\
\hline
\end{tabular}

\section{Conclusion}

Cette synthèse bibliographique a permis de mettre en évidence l'état de pollution des différentes sources d'approvisionnement en eau dans quelques milieux déshérités des villes de l'Afrique. Dans leur grande majorité, les eaux utilisées par la population sont les eaux de puits, eaux de forages et l'eau de réseau public. Ces eaux sont confrontées à la contamination microbiologique et chimique. De toutes les catégories d'eaux exploitées par les populations urbaines, les eaux de puits sont plus exposées aux problèmes de contamination. Les Facteurs de pollution sont d'origines anthropiques ou naturelles. L'état de pollution des eaux varie d'une région à une autre. Les informations collectées constituent une base pour une meilleure orientation des travaux de recherche sur les facteurs de pollution des eaux à usages domestique et les moyens de remédiation dans un cadre spécifique. Cette synthèse bibliographique aboutira à identifier le niveau de pollution des eaux à usages domestiques et les facteurs impliqués au sein des populations défavorisées de la ville de Cotonou (Bénin).

\section{CONFLIT D'INTERETS}

Les auteurs ne déclarent aucun conflit d'intérêt.

\section{CONTRIBUTIONS DES AUTEURS}

Les auteurs: EOH, MADT, NCK, EWV, GAM et EA ont tous participé à la rédaction $\mathrm{du}$ document (structuration des informations et la synthèse des données) après que la collecte des données ait été faite par $\mathrm{EOH}$.

\section{REFERENCES}

Agassounon Djikpo Tchibozo M, Ayi-Fanou L, Lozes E, Fadonougbo R, Anago GDJ, Agbangla C, Ahanhanzo C. 2012. Impacts sanitaires liés à l'usage des eaux de puits, à l'assainissement et à 
l'aménagement à Gbôdjê dans l'arrondissement de Godomey au Bénin. Int. J. Biol. Chem. Sci., 6(2): 592-602.

Ahonon A. 2011. Evaluation de la qualité physico-chimique et bactériologique des eaux de surface dans les zones montagneuses du sud-ouest du Togo : cas du canton de Lavie. Mémoire présenté en vue de l'obtention du diplôme de Master international en Environnement Eau et Santé, Université De Lome, p. 35.

Ahoudi H, Gnandi K, Tanouayi G, OuroSama K. 2015. Caractérisation PhysicoChimique et Etat de pollution par les élements traces métalliques des eaux souterraines de Lomé (Sud Togo) : cas du quartier Agoe Zongo. Larhyss Journal, 24: 41-56.

Aka N, Bamba SB, 1soro G, 1soro N. 2013. Étude Hydrochimique et microbiologique des nappes d'altérites sous climat tropical humide : Cas du Département d'Abengourou (Sud-Est de la Côte D'ivoire). Larhyss Journal, 16: 31-52.

Alia RH. 2008. les Nations Unies et le problème de l'eau dans le monde: essai sur ONU-eau. Mémoire de la maîtrise en science politique, Université du Québec, Montréal, p. 113.

Andzi Barhé T, Bouaka F. 2013. Caractérisation Physicochimique et Chloration des Eaux de Puits Consommées dans la Ville de Brazzaville-Congo. J. Mater. Environ. Sci., 4(5): 605-612.

Association Santé Environnement France, 2010. Pollution de l'eau : Origines et impacts, 5p.

Aubry P, Gaüzère B-A. 2012. Les maladies liées à l'eau, Diplôme de Médecine Tropicale des pays de l'Océan Indien, Actualités 2011, p. 7.

Bahir M, Mennani A, Jalal M, Fakir Y. 2002. Impact de la sécheresse sur les potentialités hydriques de la nappe alimentant en eau potable la ville
d'Essaouira (Mogador, Maroc). Notes méthodologiques. Science et Changements Planétaires/Sécheresse, 13-19.

Benajiba MH, Saoud Y, Lamribah A, Ahrikat M, Amajoud N, Ouled-Zian O. 2013. Evaluation de la qualité microbienne des eaux de la nappe phréatique de Martil au Maroc. Revue des Sciences de l'Eau/ Journal of Water Science, 26(3): 223233.

Botta A, Bellon L. 2004. Pollution chimique de l'eau et santé humaine. Service de médecine et santé au travail. Laboratoire de biolgénotoxicologie et mutagenèse environnementale (EA 1784, IFR PMSE 112), p. 23.

Boubakar Hassane A. 2010. Aquifères superficiels et profonds et pollution urbaine en Afrique : Cas de la communauté urbaine de Niamey (NIGER), Thèse de l'Université, Abdou Moumouni de Niamey (Niger), p. 198.

Bricha S, Ounine K, Oulkheir S, EL Haloui N, Attarassi B. 2007. Etude de la qualité physico-chimique et bactériologique de la nappe phréatique M'nasra (Maroc). Revue Afrique Science, 03(3): 391-404.

Burnol A, Duro L, Grive M. 2006. Eléments traces métalliques. Guide méthodologique, recommandations pour la modélisation des transferts des éléments traces métalliques dans les sols et les eaux souterraines. Rapport final, $\mathrm{N}^{\circ}$ INERIS-DRC-06-66246/DESP-R01a, 2354.

Chippaux JP, Houssier S, Gross P, Bouvier C, Brissaud F. 2002. Etude de la pollution de l'eau souterraine de la ville de Niamey, Niger. Bull. Soc. Pathol. Exot, 95(2): 119-223.

Coulibaly k. 2005. Etude de la qualité physico-chimique et bactériologique de l'eau des puits de certains quartiers du district de Bamako. Thèse présentée pour obtenir le grade de docteur en pharmacie, 
Faculté de Médecine de Pharmacie et d'Odonto-stomatologie Université de Bamako, p. 52.

Coulibaly L, Diomandé D, Coulibaly A, Gourène G. 2004. Utilisation des ressources en eaux, assainissement et risques sanitaires dans les quartiers précaires de la commune de Port-Bouët (Abidjan; Côte d'Ivoire). VertigO - la revue électronique en sciences de l'environnement, $\mathbf{5}$ :(3).

Dégbey C, Makoutodé M, Ouendo EM, De Brouwer C. 2010. Pollution physicochimique et microbiologique de l'eau des puits dans la Commune d'Abomey-Calavi au Bénin en 2009. Int. J. Biol. Chem. Sci., 4(6): 2257-2271.

Djuikom E, Temgoua E, Jugnia L, Nola M, Baane M. 2009. Pollution bactériologique des puits d'eau utilisés par les populations dans la Communauté Urbaine de Douala Cameroun. Int. J. Biol. Chem. Sci., 3(5): 967-978.

Dovonou FE. 2012. Diagnostic qualitatif et environnemental de l'aquifère superficiel du champ de captage intensif de Godomey au Bénin (Afrique de l'Ouest) : éléments pour un plan d'actions stratégiques de protection des ressources en eau souterrraine exploitees. Doctorat ès Science en Hydrologie et Gestion Intégrée des Ressources en Eau. Université d'Abomey, Bénin, p.143.

El Ouedghiri K, El Oualti A, El Ouchy M, Zerrouq F, Ouazzani Chahdi F, El Ouali Lalami A. 2014. Risques sanitaires liés aux composés chimiques contenus dans l'eau de boisson dans la ville de Fès : Cas des ions nitrates et nitrites). J. Mater. Environ. Sci., 5 (S1): 2284-2292.

Er-Raioui H, Bouzid S, Khannous S, Zouag MA. 2011. Contamination des eaux souterraines par le lixiviat des décharges publiques: Cas de la nappe phréatique R'Mel (Province de Larache - Maroc
Nord-Occidental). Int. J. Biol. Chem. Sci., 5(3): 1118-1134.

Fatombi JK, Avocanh G, Topanou N, Aminou T, Josse ERG. 2013. Elimination $\mathrm{du}$ fer et du manganèse d'une eau de surface par les graines de Moringa oleifera. Int. J. Biol. Chem. Sci., 7(3): 1379-1391.

Festy B, Hartemann P, Ledrans M, Levallois P, Payment P, Tricard D. 2003. Qualité de l'eau. In Environnement et santé publique-Fondement et pratiques, 333368.

Garcia-Armisen T. 2006. Etude de la dynamique des Escherichia coli dans les rivières du bassin de la Seine. Thèse de doctorat, Université Libre de Bruxelles, $81 \mathrm{p}$.

Gauthier F. 2002. Biofilms et qualité biologique de l'eau potable au cours de distribution. Mémoire de DESS Qualité et Gestion de l'Eau, p. 78.

Gendron A, Burelle S, Roy G. 2007. État de situation des rejets anthropiques de mercure dans l'environnement au Québec, Direction des politiques en milieu terrestre, p. 35.

Ghazali D, Zaid A. 2013. Etude de la qualité physico-chimique et bactériologique des eaux de la source Ain Salama-Jerri (Region de Meknes -Maroc). Larhyss Journal, 12: 25-36.

Givord L, Dorioz JM. 2010. La survie des microorganismes d'origine fécale dans les effluents et les sols. INRA thonon Umr carrtel. Etude réalisée dans le cadre du projet CasDAR Territ'Eau, 23p.

Guergazi S, Achour S. 2005. Caractéristiques physico-chimiques des eaux d'alimentation de la ville de Biskra. Pratique de la chloration. Courrier $d u$ Savoir, 6: 53.

Hartemann P. 2013. Eau de consommation, risque, santé. Sciences Eaux \& Territoires, 10 : 14-21. 
Host S, Telle-Lamberton M. 2014. Le milieu urbain, réceptacle de nuisances multiples, Territoires, incubateurs de santé ? Les Cahiers de l'IAU îdF, 170-171: 36-39.

Hoteyi SMI, Gnimadi CC, Adjadji GV, Igoué AM, Mensah GA. 2014. Analyse des risques de consommation des eaux en sachet pour les populations dans ville de Porto-Novo au Sud-Bénin. Bulletin de la Recherche Agronomique du Bénin (BRAB). Numéro spécial Agrobiodiversité et Santé publique, p. 8.

Hounsinou P, Mama D, Dovonou F. Alasane A. 2015. Seasonal evolution of the quality microbiological of the natural waters in the township of Abomey-Calavi (South Benin). British Journal of Earth Sciences Research, 3(1): 30-41.

INSPQ (Institut National de la Santé Publique du Québec). 2003. Fiche sur le plomb du Groupe scientifique sur l'eau. Institut national de santé publique du Québec, $\mathrm{p}$. 14.

Jourdan B, Piantone P, Lerouge C, Guyonnet D. 2005. Atténuation des métaux à l'aval de sites de stockage de déchets (Synthèse bibliographique). BRGM/RP 54417, p. 110.

Kaboré A, Savadogo B, Rosillon F, Traoré AS, Dianou D.2013. Optimisation de l'efficacité des graines de Moringa oleifera dans le traitement des eaux de consommation en Afrique sub-saharienne : cas des eaux du burkina faso. Revue des Sciences de l'Eau, 26(3): 209-220.

Kenfaoui A. 2008. Economisons l'eau en la préservant de la pollution. Revue HTE, 140: 94-96.

Lagnika M, Ibikounle M, Montcho J-PC, Wotto VD, Sakiti NG, 2014. Caractéristiques physico-chimique de l'eau de puits dans la commune de Pobè, Bénin. Journal of Applied Biosciences, 79: 6887-6897.

Lalanne F. 2012. Etude de la qualité de l'eau le long de la chaîne approvisionnement au niveau des consommateurs dans 10 villages de la Province du Ganzourgou, (Région du Plateau Central, Burkina Faso), p. 71.

Laurent F. 2013. Agriculture et pollution de l'eau: modélisation des processus et analyse des dynamiques territoriales. Sciences de l'environnement, Université du Maine, p. 188.

Lavie E, El-Tayib N. 2014. Du robinet au consommateur : qualité de l'eau potable dans le contexte domestique de l'agglomération de Khartoum, Soudan, Cybergeo : European Journal of Geography, Espace, Société, Territoire, document 664.

Levallois P, Phaneuf D. 1994. La contamination de l'eau potable par les nitrates : analyse des risques à la santé. Revue Canadienne de Santé Publique, 85(3) : 192-196.

Mahamat SAM, Maoudombaye T, Abdelsalam T, Ndoumtamia $G$, Loukhman B. 2015. Évaluation de la qualité physico-chimique des eaux d'adduction publique de la Société Tchadienne des Eaux à N'djamena au Tchad. Journal of Applied Biosciences, 95: 8973-8980.

Matini L, Moutou JM, Kongo-Mantono MS. 2009. Evaluation hydrochimique des eaux souterraines en milieu urbain au SudOuest de Brazzaville, Congo. Afrique Science, 5(1): 82-98.

Mbawala A Abdou, Ngassoum M-B. 2010. Evaluation de la pollution physicochimique et microbienne des eaux de puits de Dang-Ngaoundéré (Cameroun). Int. J. Biol. Chem. Sci., 4(6): 1962-1975.

Mfonka Z, Ndam Ngoupayou JR, Ndjigui PD, Zammouri M, Kpoumie A, Rasolomanana E. 2015. Hydrochimie et potabilité des eaux du bassin versant du Nchi dans le plateau Bamoun (Ouest Cameroun). Int. J. Biol. Chem. Sci., 9(4): 2200-2218. 
Molinie L. 2009. Dispositifs rustiques d'alimentation et de traitement de l'eau potable pour des services de petites tailles en régions défavorisées. Synthèse Technique. Agro Paris Tech - Engref, p. 28.

Mpakam HG, Kamgang Kabeyene BV, Kouam Kenmogne GR, Bemmo N, Ekodeck GE. 2006. L'accès à l'eau potable et à l'assainissement dans les villes des pays en développement (cas de Bafoussam au Cameroun). Vertigo-Revue en Sciences de l'Environnement, 7(2).

Mpakam HG, Kouam Kenmogne GR, Tamo Tatietse T, Maire E, Boeglin JL, Ekodeck GE, Dupre B. 2009. Etude des facteurs de pollution des ressources en eau en milieu urbain : cas de Bafoussam (OuestCameroun). Actes du colloque international sur le thème « changements climatiques et évaluation environnementale », Niamey (Niger) du 26 au 29 Mai 2009, p. 27.

Mulliss RM, Revitt DM, Shutes RBE. 1997. The impacts of discharges from two combined sewer overflows on the water quality of an urban watercourse. Water Sci. Technol., 36: 195-199.

Nanga N, Ngene JP, Tsala DE, Ngoule C, Lamare N. 2014. Relation between spring and well water pollution and diarrheal diseases in the Centre Hospitalier Dominicain Saint Martin de Porres (CHDSMP) of Mvog -Betsi neighborhood. Health Sci. Dis., 15(3): 18.

Ndiaye P, Badu IO, Dieng M, Fall C, Diadu AT. 2010. Qualité de l'eau de consommation des ménages: analyse et plan d'action en zone rurale sénégalaise. Santé Publique, 22(2): 193-200.

Ngnikam E, Mougoue B, Tietche F. 2007. Eau, Assainissement et impact sur la santé : étude de cas d'un écosystème urbain à Yaoundé. Actes des JSIRAUF, p. 13.
Nguimal C-R et, Balikouzou-Hinna DA, Gothard-Bassebe M-CR et Semballa S. 2005. Gestion de la qualité de l'eau, conflits et risques dans la ville de Bangui (République centrafricaine). Géocarrefour, 80(4): 325-334.

Nkurunziza G. 2013. Etude de la qualité de l'eau de boisson le long de la chaîne de l'eau dans les ménages de trois régions du Burkina Faso: cas des villages de Kamboinsé, Koubri, Sikoro et Bapla. Mémoire pour l'obtention du master en ingénierie de l'eau et de l'environnement, p. 84 .

Nola M, Njiné T, Kemka N, Zébazé Togouet $\mathrm{SH}$, Servais P, Messouli M, Boutin Cl, Monkiedje A et Foto Menbohan S. 2006. Transfert des bactéries fécales vers une nappe phréatique à travers une colonne de sol en région équatoriale : influence de la charge en eau appliquée en surface. Revue des Sciences de l'Eau / Journal of Water Science, 19(2): 101-112. DOI: http://dx.doi.org/10.7202/013044ar

Odoulami L. 2009. La problématique de l'eau potable et la santé humaine dans la ville de Cotonou (République du Bénin). Thèse de Doctorat, Université d'Abomey-Calavi, Géographie et Gestion de l'Environnement, p. 230.

OMS (Organisation Mondiale de la Santé). 1997: Guideline for Drinking Water Quality 2nd Edition. Volume 3, Surveillance and Control of Community Supplies, Geneva.

OMS/UNICEF (Organisation Mondiale de la Santé/Fond des Nations unies pour l'enfance). 2008. Un Aperçu de la situation de l'eau potable en Afrique.11ème Sommet des Chefs d'Etat et de Gouvernement de l' l'Union Africaine sur le thème : réalisation des Objectifs du Millénaire concernant l'Eau et l'Assainissement, p. 13.

Ouhsassi M, Khay EO, Amajoud N, Abrini J. 2013. Etude de la qualité 
microbiologique des eaux Skundo, Ancien Réseau d'approvisionnement en eau (Medina de Tetouan, Maroc). Sciencelib., 5(130211) :17.

Payment P, Hartemann P. 1998. Les contaminants de l'eau et leurs effets sur la santé. Revue des sciences de l'eau / Journal of Water Science, 11: 199-210.

PNUD (Programme des Nations Unies pour le Développement). 2006. Rapport mondial sur le développement humain (PNUD) 2006. Au-delà de la pénurie : Pouvoir, pauvreté et la crise mondiale de l'eau, $p$. 422.

Poisson A. 2011. Désinfection dans des réseaux de distribution d'eau potable : efficacité de traitements sur des biofilms et importance des méthodes d'évaluation. Anses. Bulletin de Veille Scientifique. Santé / Environnement / Travail, 13: 5355.

pS-Eau (Programme Solidarité Eau). 2012. Accès à l'eau potable dans les pays en développement, 18 questions pour des services durables, Guide pratique, p. 50.

Rodier J, Legube B, Merlet N. 2009. L'Analyse de l'Eau, éd DUNOD: 749775.

Rosillon F. 2003. Introduction à la microbiologie des eaux domestiques. Stage intensif de recyclage sur les eaux usées domestiques et industrielles, Direction de l'Hygiène et de l'Assainissement de Base (DHAB). Fondation Universitaire Luxembourgeoise 6700 Arlon (Belgique), p. 26.

Sackou Kouakou JG, Oga S, Claon S, Bama M, Koua MD, Houénou Y, Kouakou KL. 2010. Conditions d'accès et de stockage de l'eau : enquête dans les ménages en zone périurbaine à Abidjan. Santé Publique, 2(24):133-142.

Servais P, Billen G, Garcia-Armisen T, George I, Goncalvez A., Thibert S. 2009. La contamination microbienne dans le bassin de la Seine. Edition. Agence de l'Eau Seine Normandie, p. 49.

Soncy K, Djeri B, Anani K, Eklou-Lawson M, Adjrah Y, Karou DS, Ameyapoh Y, de Souza C. 2015. Évaluation de la qualité bactériologique des eaux de puits et de forage à Lomé, Togo. Journal of Applied Biosciences, 91: 8464 - 8469.

Soro N, Ouattara L, Dongo K, Kouadio E K, Ahoussi EK, Soro G, Oga M-S, Savane I, Biemi J. 2010. Déchets municipaux dans le District d'Abidjan en Côte d'Ivoire: sources potentielles de pollution des eaux souterraines. Int. J. Biol. Chem. Sci., 4(6): 2203-2219.

St-Laurent J, Levallois P, Gauvin D, Courteau M. 2013. Sources résidentielles de plomb et niveaux de plombémie chez de jeunes enfants habitant d'anciens arrondissements de Montréal, Québec, $77 \mathrm{p}$.

Sy I, Koita M, Traoré D, Keita M, Lo B., Tanne M, Cissé G. 2011. Vulnérabilité sanitaire et environnementale dans les quartiers défavorisés de Nouakchott (Mauritanie) : analyse des conditions d'émergence et de développement des maladies en milieu urbain sahélien. [VertigO] Revue en sciences de l'Environnement, 11(2): 17p.

Temgoua E. 2011. Chemical and Bacteriological Analysis of Drinking Water from Alternative Sources in the Dschang Municipality, Cameroon. Journal of Environmental Protection, 2: 620-628.

Totin H, Boko M, Edorh P. 2008. Groundwater pollution in shantytowns of Cotonou: which strategies to limit waterborne diseases risks? Poster, Laboratory of Climate, Water Resources and Ecosystem Dynamic Research, University of Abomey-Calavi Republic of Bénin.

UNICEF (Fond des Nations unies pour l'enfance). 2011. Etude sur la qualité 
microbiologique de l'eau en Mauritanie, $66 \mathrm{p}$.

Van der Werf H. 1996. Assessing the impact of the pesticides on the environnement. Agriculture, Ecosystems and Environment, 60: 81-96.

Vaute L. 1999. Identification d'éléments indésirables ou toxiques dans les ressources en eau potentielles du bassin ferrifère lorrain. Rapport BRGM R 40908, p. 37.

Viraraghavan T, Subramanian KS, Venkata Rao B. 1999. Impact of household plumbing fixtures on drinking water quality, a review, Intern. J. Environ. Studies, 56: 717-743.

Worm J, Hattum Tv. 2006. La collecte de l'eau de pluie à usage domestique. Agrodok 43, p. 86.

Yapo OB, Mambo V, Seka, A, Ohou MJA, Konan F, Gouzile V, Tidou AS, Kouamé
KV, Houénou P. 2010. Evaluation de la qualité des eaux de puits à usage domestique dans les quartiers défavorisés quatre communes d'Abidjan (Côte d'Ivoire) : Koumassi, Marcory, Portbouet et Treichville. Int. J. Biol. Chem. Sci., 4(2): 289-307.

Yélognissè CLHH. 2007. Amélioration des conditions d'accès à une eau potable pour l'eau de boisson dans les milieux ruraux du bénin: étude des pratiques locales. Maîtrise en sciences de l'environnement, Université du Québec à Montréal, p. 117.

Zerhouni J, Rhazi Filali F, Aboulkacem A. 2015. Qualité et facteurs de risque de pollution des eaux souterraines périurbaines de la ville de Sebaa Ayoune (Meknes, Maroc). Larhyss Journal, 22: 91-107. 